\title{
Microstructural Analysis with Graded and Non-Graded Indium in InGaN Solar Cell
}

\author{
Illknur Kars Durukan ${ }^{1, *}$, Mustafa Kemal Öztürk ${ }^{2,3}$, Süleyman Çörekçii ${ }^{4}$, Mehmet Tamer ${ }^{3,7}$, \\ Yunus Baş ${ }^{5}$, Süleyman Özçelik ${ }^{2,3}$, and Ekmel Özbay ${ }^{6}$
}

In this study are graded and non graded InGaN/GaN samples grown on c-oriented sapphire substrate using the Metal Organic Chemical Vapour Deposition (MOCVD) technique. The structural and morphological properties of the grown InGaN/GaN solar cell structures are analyzed using High Resolution X-ray Diffraction (HRXRD), atomic force microscopy (AFM). Each structures $c$ and a lattice parameters strain, biaxial strain, hydrostatic strain, stress, lattice relax, tilt angle, mosaic crystal size, dislocation densities of $\mathrm{GaN}$ and InGaN layers are determined by XRD measurements. In accordance with these calculations, the effect of graded structure on the defects, are discussed. As a dramatic result; although values of full width at half maximum (FWHM) are broad, a considerable decrease at dislocations is noticed. The AFM observations have revealed that the two dimensional growth of the graded sample is more significant and its roughness value is lower. JV measurements shown that the performance of the graded structure is higher. It is determined that all test results are consistent with each other.

Keywords: InGaN/GaN Solar Cell, MOCVD, HRXRD, AFM.

\section{INTRODUCTION}

Recently, group III-nitride wide semiconductors band gap has led to the development of light emitting devices and high efficiency solar cells. ${ }^{1-5}$ Furthermore, InGaN alloys have become subject to an increasing research effort because of its high carrier mobility, high drift velocity, and durability in high-temperature and radiation environments. All of these are necessary to produce high efficiency solar cells. ${ }^{6-10}$ However, the growth of a high crystal quality $\mathrm{GaN} / \mathrm{InGaN}$ solar cell structure is difficult because of the large difference in the

${ }^{1}$ Life Sciences Research and Application Center, Gazi University, Gölbaşı, Ankara, Turkey

${ }^{2}$ Department of Physics, Faculty of Sciences, Gazi University, Ankara, Turkey

${ }^{3}$ Photonics Research Center, Gazi University, 06500 Ankara, Turkey

${ }^{4}$ Energy Systems Engineering Department, Faculty of Technology, Kırklareli University, Kırklareli, Turkey

${ }^{5}$ National Boron Research Institute, 06520, Ankara, Turkey

${ }^{6}$ Department of Physics, Bilkent University 06800, Bilkent, Ankara, Turkey

${ }^{7}$ Department of Physics, Zirve University, Gaziantep, Turkey

*Author to whom correspondence should be addressed.

Email: ilknurdurukan@gazi.edu.tr

Received: 18 March 2016

Accepted: 18 May 2016 suitable coefficient of thermal expansion (28\% GaN and $\mathrm{InN})$ and the lattice disconformity $(10.6 \% \mathrm{GaN}$ and $\mathrm{InN})$ between films and substrate. For the III-nitrides, especially InGaN grown on a GaN template, graded buffer layer is used to improve the quality of the epilayer. However, there are only a few reports researching the continuously graded buffer layer ${ }^{11.12}$ and thus this buffer layer technique has not been accurately understood. The purpose of this study is to report the results for In graded $\mathrm{In}_{x} \mathrm{Ga}_{1-x} \mathrm{~N}$ $(10.5 \leq x \leq 18.4)$ and non-graded $\operatorname{In}_{x} \mathrm{Ga}_{1-x} \mathrm{~N}(13.6 \leq$ $x \leq 24.9)$ epitaxial structures obtained by using metal organic chemical vapor deposition (MOCVD). We have investigated the graded interlayer technique for improving of the InGaN epilayer quality on sapphire substrates. Our study found surface and volume defects such as strain, hydrostatic and biaxial strain components, stress, the plane tilt angle, mosaic crystal size, heterogeneous strain, dislocation densities and surface morphology via mosaic crystal model. In addition, the effects of graded layers on different type of dislocation densities are discussed with XRD measurements. The atomic force microscope (AFM) has been used for observing the surface morphologies of the films and their roughness. Further, Current density, potential $(J V)$ measurements and the solar battery performance of the grown structures are discussed. 


\section{GROWTH CONDITIONS}

In the present study, InGaN/GaN solar cell structures (Fig. 1) were grown via the MOCVD technique on $c$-oriented sapphire substrates. When sample A was deposited as graded, sample B was deposited as nongraded. Prior to the growing process, substrates were cleaned under $\mathrm{H}_{2}$ gas to eliminate the contaminations on the surface. After the decontamination procedure, for each sample, a GaN nucleation layer was grown. The thickness of this nucleation layer was $10 \mathrm{~nm}$. Following the completion of the nucleation layer growth, the GaN buffer layer was grown. The thickness of the GaN buffer layer was $1.6 \mu \mathrm{m}$. The growing process was endured by releasing the $\mathrm{SiH}_{4}$ source and an $n$-type $\mathrm{GaN}$ layer was obtained with the aid of the $\mathrm{SiH}_{4}$ source. Furthermore, the flow rate of the $\mathrm{SiH}_{4}$ source was $10 \mathrm{sccm}$. The $\mathrm{In}_{x} \mathrm{Ga}_{1-x} \mathrm{~N}$ active layers were grown for samples $\mathrm{A}$ and $\mathrm{B}$ at temperatures between $745-760{ }^{\circ} \mathrm{C}$ degree and an In flow rate of $75 \mathrm{sccm}$. While the InGaN layer thicknesses in sample A were $25 \mathrm{~nm}$ (graded) and $200 \mathrm{~nm}$ (graded), the InGaN layer (non-graded) had a thickness of $220 \mathrm{~nm}$. The active layers were grown between $1.9 \mu \mathrm{m}$ thick $n$-type $\mathrm{GaN}$ and p-type InGaN contact layers.

The $\mathrm{p}^{+}$InGaN layer was grown by using a $\mathrm{Mg}$ doping source. The $\mathrm{Mg}$ flow rate was adjusted to $40 \mathrm{sccm}$ to provide more doping. The InGaN layer was converted to a graded status by keeping the InGaN layers's in flow rate continuous.

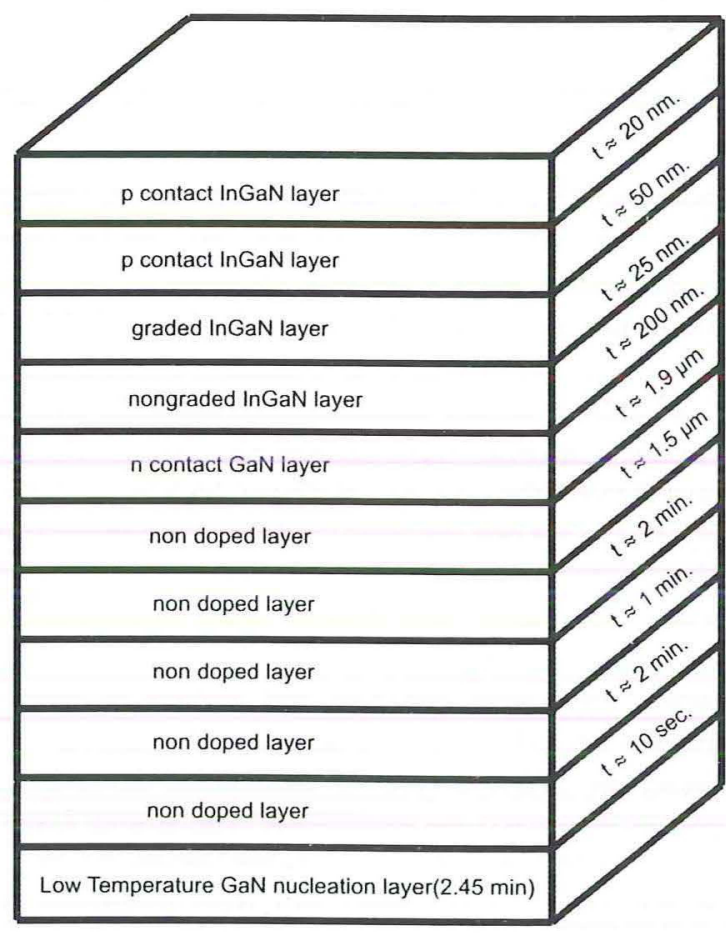

Sample A

\section{RESULTS AND DISCUSSION}

\subsection{XRD Analysis}

Omega curves of samples $\mathrm{A}$ and $\mathrm{B}$ at a wide angle and diffraction peaks of InGaN and $\mathrm{GaN}$ for all symmetric diffraction planes are defined (Fig. 2). The layers of InGaN and $\mathrm{GaN}$ are repeated as triple peaks (or sets). These peaks match up to (002), (004) and (006) symmetric planes of hexagonal crystal structure, respectively. For both samples, the peaks of GaN coincide with those of in case of strainfree. Besides, low severe azimuth peaks belonging to $\mathrm{GaN}$ and $\mathrm{Al}_{2} \mathrm{O}_{3}$ can be observed. The peaks with high intensity and low FWHM of InGaN show that they are of high quality. For sample A, the peaks show continuity depending on In ratio of graded InGaN layer (Fig. 2 inset). For sample $\mathrm{B}$, two different peaks as $x_{1}$ and $x_{2}$ alloy rates of InGaN are observed depending on the rate of In. On the other hand, X-ray diffraction peak from asymmetric planes (105) is examined to investigate quality of the crystal structure (Fig. 3). In addition, peak of In graded InGaN and two peaks of In non-graded InGaN are observed. The (004) peak for every two samples is separated with InGaN's two omega peaks in the inset of Figure 3. High intensity and lower FWHM values verify that the crystal quality is high (Table I). Otherwise, the asymmetric peak positions and FWHM are used for calculations of lattice parameter and mosaic defects of hexagonal structure.

Nitrogenous compounds and alloys like GaN, InGaN are in hexagonal structures. The crystal quality decreases

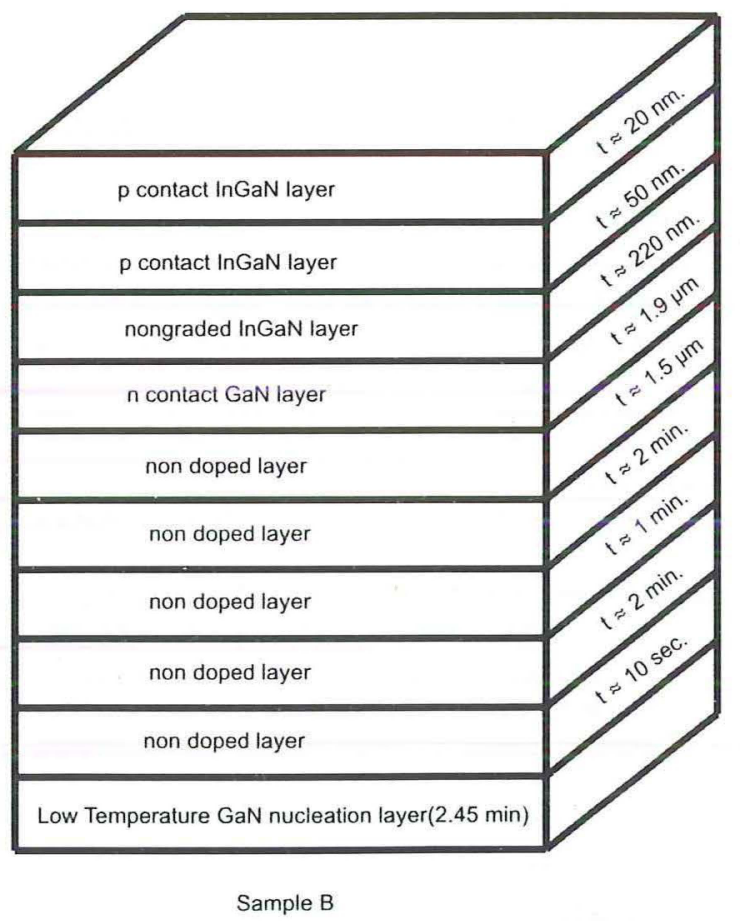

Fig. 1. InGaN layered solar cell structure (a) In graded sample A, (b) Non In graded sample B. 


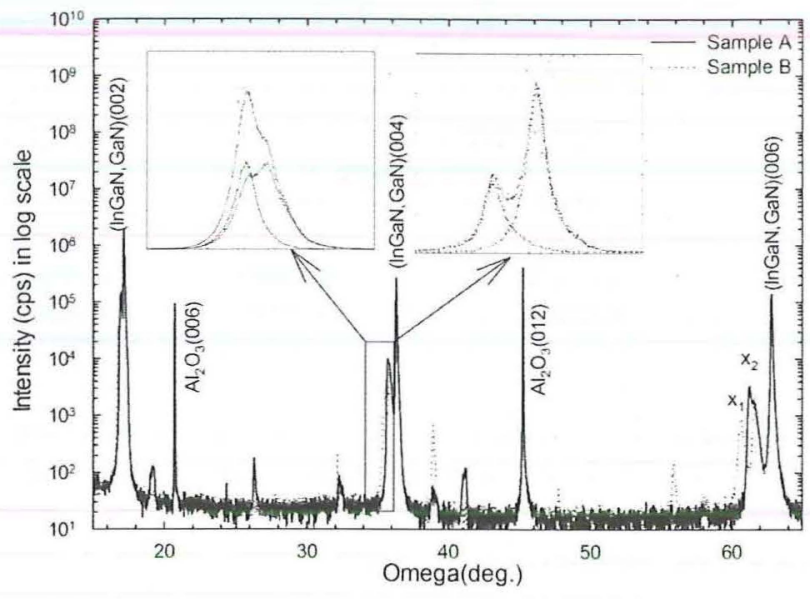

Fig. 2. Omega curves of samples $A$ and $B$ at a wide angle for symmetric planes.

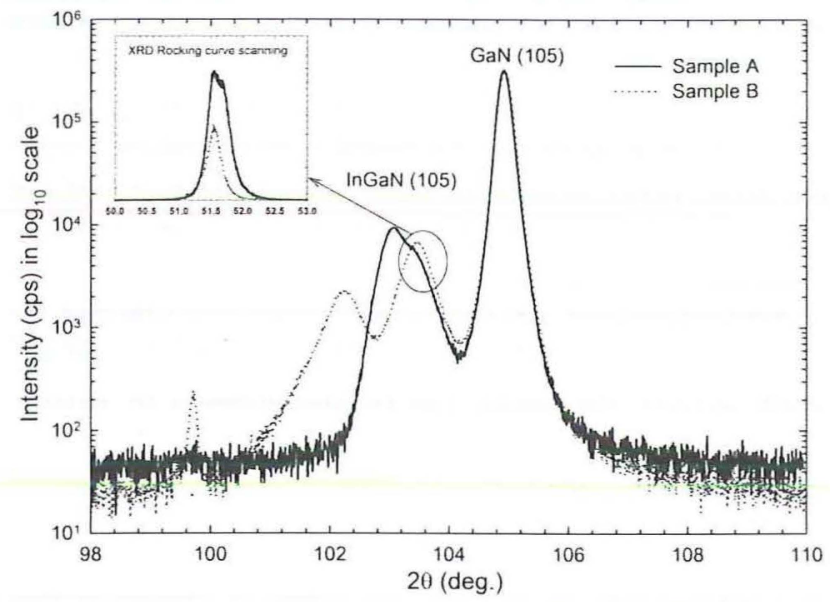

Fig. 3. $2 \theta$ curves of samples A and B at wide angle for (105) planes.

when the symmetric peaks are broadened. The broadening of the symmetric and asymmetric peaks for these types of layers are noticed by the change in plane tilt, twist angles and the lateral crystal sizes parallel with the substrate surface.

FWHM values of sample A except for GaN (004) value are found to be wider than those of sample B. The splitting of sample B cannot be observed because sample A has graded structure (Fig. 2). Therefore, a large amount out of the FWHM value in sample A depends on the constant of In ratio. For example, this FWHM value is the 890.64 arcsec unit and belongs to InGaN (004) for sample A, which is the expected value according to InGaN structure.
According to the Vegard's law, the ratio of the alloy in InGaN layer depends on the peak positions of $\mathrm{InN}$ and $\mathrm{GaN}$. When the $x$ value of InGaN is different from what it is supposed to be, the Vegard's law is used for calculation. However, to differentiate the strain value appendage of InGaN at $x$ value, the cubical equation of $A x^{3}+B x^{2}+$ $C x+D=0$ can be used. ${ }^{13} A, B, C$ and $D$ coefficients of this cubical equation includes $\mathrm{InN}$, universal $a$ and $c$ lattice parameters of $\mathrm{GaN}$ and Poission ratios. $x$ ratio can be solved using a program in $0-1$ interval, and qualities such as strain and relax are calculated, afterwards.

Ge $022(+,-,-,+)$ monochromator and Goebbels mirror were employed to separate $k \alpha_{1}$ and $k \alpha_{2}$ in the parallel sheaf and to eliminate $k \alpha_{2}$ in the HRXRD technique. The measurements of the symmetrical and asymmetrical reflections, Bragg angle $\theta$ and lattice curving angle $\tau$ can be calculated through the $\theta=$ $\left(\theta_{+}+\theta_{-}\right) / 2$ and $\tau=\left(\theta_{+}+\theta_{-}\right) / 2$ equations. ${ }^{14} a$ and $c$ lattice parameters of corrupted InGaN hexagonal unit cell are calculated from a proper angle of the $h k(-h-k) l$ reflection. Three different techniques could be used to calculate the lattice parameters. For the first technique, the lattice parameter of the InGaN, by taking the Vegard's law into consideration, is found by adding $x$ numbered $\mathrm{InN}$ lattice parameters to $(1-x)$ numbered GaN lattice parameters. To obtain $a$ and $c$ lattice parameters on the asymmetrical planes, by means of using $\sin \tau$ and $\cos \tau$ at the denominator of the equations below, the second technique can be procured. ${ }^{15}$

$$
\begin{gathered}
c=\frac{\lambda l}{2 \sin \theta \cos \tau} \\
a=\frac{\lambda \sqrt{4 / 3} \sqrt{h^{2}+h k+k^{2}}}{2 \sin \theta \sin \tau}
\end{gathered}
$$

For the last technique, using the $\left(1-D \cos ^{2} \theta\right) /(r \sin \theta)$ error function and Bragg Law, the parameters could be calculated by;

$$
\begin{aligned}
c & =\frac{l \lambda}{2 \sin \theta} x \quad \text { (error function) } \\
a^{(h k l)} & =c d_{h k l} \sqrt{\frac{(4 / 3)\left(h^{2}+k^{2}+h k\right)}{c^{2}-l^{2} d_{h k l}^{2}}}
\end{aligned}
$$

Equations (3) and (4). ${ }^{16}$ On the error function, $D$ is the possible displacement of the sample with goniometer axis on asymmetrical plane (equatorial plane), and its value is 0.02 . $R$, which is the distance of the sample from the detector $(450 \mathrm{~mm})$.

The lattice parameters of InGaN graded solar cell (sample A) are given as value ranges (Table II). The reason for

Table I. Peak broadening of (004), (105) diffraction planes of $\mathrm{InGaN}$ and $\mathrm{GaN}$ in arcsec unit.

\begin{tabular}{lcccc}
\hline Sample & InGaN $(004)$ & InGaN (105) & GaN (004) & GaN (105) \\
\hline A & 890.64 & 2271.96 & 285.12 & 859.68 \\
B & $(550.08,542.52)$ & $(1679.40,1606.30)$ & 300.60 & 804.24 \\
\hline
\end{tabular}


Table II. The $c$ - and $a$-lattice parameters found through Vegard's law, Vegard's law with cubic equation and error function on InGaN layer(s) for all samples and their comparisons.

\begin{tabular}{|c|c|c|c|c|c|c|}
\hline Sample & $\begin{array}{c}C_{\text {vegard's law with cubic equation }} \\
(\mathrm{nm})\end{array}$ & $\begin{array}{l}c_{\text {eq. } 1} \\
(\mathrm{~nm})\end{array}$ & $\begin{array}{l}c_{\mathrm{eq} .3} \\
(\mathrm{~nm})\end{array}$ & $\begin{array}{l}A_{\text {vegard's slaw with cubic equation }} \\
(\mathrm{nm})\end{array}$ & $\begin{array}{l}A_{\text {eq. } 2} \\
(\mathrm{~nm})\end{array}$ & $\begin{array}{l}A_{\text {eq. } 4} \\
(\mathrm{~nm})\end{array}$ \\
\hline A & $\begin{array}{l}0.52813- \\
0.52619\end{array}$ & $\begin{array}{l}0.52748- \\
0.52567\end{array}$ & $\begin{array}{l}0.52813- \\
0.52619\end{array}$ & $\begin{array}{l}0.32302- \\
0.32218\end{array}$ & $\begin{array}{l}0.32445- \\
0.32332\end{array}$ & $\begin{array}{l}0.32118- \\
0.32261\end{array}$ \\
\hline B & $\begin{array}{l}0.53130 \\
0.52615\end{array}$ & $\begin{array}{l}0.53067 \\
0.52565\end{array}$ & $\begin{array}{l}0.53130 \\
0.52615\end{array}$ & $\begin{array}{l}0.32503 \\
0.32225\end{array}$ & $\begin{array}{l}0.3264 \\
0.32332\end{array}$ & $\begin{array}{l}0.32360 \\
0.32325\end{array}$ \\
\hline
\end{tabular}

this behavior is broadening of $\mathrm{InGaN}$ reflection as a consequence of increased In ratio. Lattice parameters are given as different two values for the non graded $\mathrm{InGaN}$ solar cell (sample B). Correction function is applied for the lattice parameters in Eqs. (1) and (2) because peak intensity observed in large Bragg angles is far away from linearity. The absolute lattice parameters were obtained using correction functions (3rd and 4th equations). Accordingly, the lattice parameters obtained with cubic equations and Vegard's law match the lattice parameters obtained by error calculations.

The point and line defects of $\mathrm{GaN}$ layer cause directional shifts on the lattice, and the shift leads to minute deviations on the peak. The distance between strain planes is directly related to the lattice parameters and to the ratio of In. Moreover, the strain risen as the polar angle increases and the square of the sinus of the angle provides a definition for the strains on the lattice. If these strains are gradient, they must have three axes, and in this case there must be a component of the surface-normal strain. A strain can be calculated using the base plate or its universal values. Here, the strain is calculated using the universal values of InGaN and $\mathrm{AlGaN}$. The strain equation can be given as

$$
\frac{\delta d}{d}, \frac{\delta a}{a}, \frac{\delta c}{c}=\delta \theta \cot \theta
$$

with its error function. ${ }^{15}$

On two dimensions, biaxial strain is known as lattice stressed or strained. Lattice strain is the sum of the biaxial strain components on two dimensions and the sum of the hydrostatic components on three dimensions. The components of biaxial and hydrostatic strain are as follows: ${ }^{17-19}$

$$
\begin{gathered}
\varepsilon_{c}=\varepsilon_{c}^{b}+\varepsilon_{h}, \quad \varepsilon_{a}=\varepsilon_{a}^{b}+\varepsilon_{h} \\
\varepsilon_{h}=\frac{c_{33} \varepsilon_{c}+2 c_{13} \varepsilon_{a}}{2 c_{13}+c_{33}}
\end{gathered}
$$

When strain values are between $(0.995-1.23) \times 10^{-3}$ ranges for sample $\mathrm{A}$, they are $(0.995-1.80) \times 10^{-3}$ ranges for sample B (Table III). The second InGaN peak of sample $\mathrm{B}$ is overlapped with the right edge of the graded InGaN peak of sample A (Fig. 2 inset). Thus, strain values are obtained as the same values. While $a$-strain values of sample A are $[(-0.441)-(-0.353)] \times 10^{-3}$ ranges, they are $[(-0.429)-(-0.331)] \times 10^{-3}$ ranges. These values show that hexagonal lattice of sample $A$ is more compressive strain. While the crystal lattice of sample $\mathrm{A}$ in $c$ direction for the two dimensional strain values is more extensional, it is more compressive in $a$ direction. Two dimensional strain values are given in Table III. It is seen that hydrostatic strain values resulting from point defects is more compressive in InGaN crystal structure. Hydrostatic strain values are given in Table III.

Alloy structures on hydrostatic strain are affected by doping and point defects. Depending on the size of the strain defects, the lattice can be compression or tensile. Different types of point defects might emerge, depending on the growth conditions. In general, it can be expected that the effective hydrostatic strain in $\mathrm{GaN}$ originates from $\mathrm{Al}_{\mathrm{Ga}}, \mathrm{N}_{\mathrm{In}}, \mathrm{Ga}_{\mathrm{In}}, \mathrm{N}_{\mathrm{Ga}}$ substitutional type point defects, $\mathrm{N}_{\mathrm{i}}$, $\mathrm{Ga}_{\mathrm{i}}, \mathrm{Al}_{\mathrm{i}}$ and In interstitial point defects, $\mathrm{V}_{\mathrm{In}}$ and $\mathrm{V}_{\mathrm{Ga}}$ vacancies Refs. [20,21] The covalent radius of the $\mathrm{Al}$ $(1.43 \AA)$ can come from $\mathrm{Al}_{2} \mathrm{O}_{3}, \mathrm{Ga}(1.22 \AA)$ and In $(1.63 \AA)$ atoms are considerably larger than the covalent radius of the $N(0.70 \AA)$ atom. Covalent values can be easily found in textbooks or in some other way. Therefore, $\mathrm{Al}_{\mathrm{Ga}}, \mathrm{Ga}_{\mathrm{N}} \mathrm{In}_{\mathrm{N}}, \mathrm{Al}_{\mathrm{N}}, \mathrm{In}_{\mathrm{Ga}}, \mathrm{In}_{\mathrm{Al}}, \mathrm{In}_{\mathrm{i}}, \mathrm{Ga}_{\mathrm{i}}, \mathrm{Al}_{\mathrm{i}}$ and $\mathrm{N}_{\mathrm{i}}$ type defects cause a crystal lattice expansion, whereas $\mathrm{Ga}_{\mathrm{Al}}$, $\mathrm{N}_{\mathrm{Ga}}, \mathrm{N}_{\mathrm{In}}, \mathrm{N}_{\mathrm{Al}}, \mathrm{Ga}_{\text {In }}, \mathrm{Al}_{\text {In }}, \mathrm{V}_{\mathrm{Al}}, \mathrm{V}_{\mathrm{Ga}}, \mathrm{V}_{\text {In }}$, and $\mathrm{V}_{\mathrm{N}}$ type point defects lead to crystal lattice compression. In our case, we find that the hydrostatic strain in first two temperature values of the InGaN layer is of compressive character and large by absolute value (Table VI). Therefore, we suppose that the relative concentrations of $\mathrm{Ga}_{\mathrm{Al}}, \mathrm{N}_{\mathrm{Ga}}, \mathrm{N}_{\mathrm{In}}, \mathrm{N}_{\mathrm{Al}}$, $\mathrm{Ga}_{\text {In }}, \mathrm{Al}_{\text {In }}, \mathrm{V}_{\mathrm{Al}}, \mathrm{V}_{\mathrm{Ga}}, \mathrm{V}_{\text {In }}$, and $\mathrm{V}_{\mathrm{N}}$ type defects are dominant in other types of point defects.

Table III. $a$ - and $c$-lattice strains, $a$ - and $c$-biaxial strains, hydrostatic strain in InGaN layers of all the samples.

\begin{tabular}{lcccr}
\hline Sample & $\begin{array}{c}c \text {-strain nm } \\
\left(\times 10^{3}\right)\end{array}$ & $\begin{array}{c}a \text {-strain nm } \\
\left(\times 10^{-3}\right)\end{array}$ & $\begin{array}{c}\text { Biaxial strain c } \\
\left(\times 10^{-3}\right)\end{array}$ & $\begin{array}{c}\text { Biaxial strain } a \\
\left(\times 10^{-3}\right)\end{array}$ \\
\hline A & $1.23-0.995$ & $(-4.41)-(-3.53)$ & $(2.47)-(1.99)$ & $(-3.18)-(-2.53)$ \\
B & $1.80,0.995$ & $(-4.29),(-3.31)$ & $(2.37),(1.87)$ & $(-3.11),(-2.38)$ \\
\hline
\end{tabular}


Table IV. Stress $x, y, z$ and stress size of InGaN.

\begin{tabular}{lllr}
\hline Sample & $x$-stress (Gpa) & $y$-stress (Gpa) & Stress size (Gpa) \\
\hline A & $(-1.33)-(1.04)$ & $(-1.35)-(1.05)$ & $(-1.32)-(-1.03)$ \\
B & $(-1.35),(0.97)$ & $(-1.37),(0.98)$ & $(-1.34),(-0.97)$ \\
\hline
\end{tabular}

On the epitaxial layer, the internal plane strain can be calculated using the equations below, if the $a$-oriented biaxial strain and the material elasticity constants are known. ${ }^{19,22}$

$$
\sigma_{f}=\left(c_{11}+c_{12}-2 \frac{c_{13}^{2}}{c_{33}}\right) \varepsilon_{a}^{b}
$$

Here, the information in the parenthesis is labeled as biaxial module $\left(M_{f}\right)$; hence, the biaxial strain is shown with $\sigma_{f}=M_{f} \varepsilon_{a}^{b}{ }^{19}$

The magnitude of stress is obtained by using stress components (Table IV). When the sizes of stress for sample A changes between (1.80-2.31) GPa ranges, the values of $(1.69,2.35) \mathrm{GPa}$ are observed for sample B. Thus, it is seen that the stress components are about same sizes for the two samples.

Strain relax is defined as the percentage of the ratio of the difference of relaxed lattice length between layers and substrate layers to the difference of universal lattice lengths, and it is represented with the equation below. ${ }^{15}$

$$
R_{\%}=\frac{a_{\|}^{L(\text { means) }}-a_{\|}^{S \text { (means) }}}{a_{\|}^{L(0)}-a_{\|}^{S(0)}} \times 100
$$

Lattice structure of sample A has more relaxation than that of sample B (Table V).

The symmetric plane is the plane which intersects the normal of the surface. Assuming that the sample plane is initially in the $z$ direction, if this plane diverges with an angle to the $z$ axis, creating a surface angle, there will be a crystal plane with observed asymmetric diffraction. The incident and reflected wave vectors used to provide the interference of these planes are to ensure the Bragg diffraction conditions. If a symmetric diffraction cause the lateral crystal size of the mosaic blocks and the plane tilt angle failure the expansion of the HRXRD twist curves which are vertical to the pole axis. ${ }^{23}$ The expansion of the reflection plane used to differentiate between both defects is defined by a linear equation. The WilliamsonHall (W-H) study can be used for this purpose. ${ }^{23}$ The W-H graphic is the FWHM graphic of the HRXRD twist curve as a function of the reflection plane level, meaning that

Table V. Lattice relax of InGaN layers in the samples.

\begin{tabular}{lc}
\hline Sample & Lattice relaxation (\%) \\
\hline A & $1.34783-1.34826$ \\
B & $1.22875,1.32033$ \\
\hline
\end{tabular}

each reflection is FWHM $(\sin \theta) / \lambda$ drawn against $(\sin \theta) / \lambda$ and the drawn curve is quitted with the linear equation. The W-H curves for the GaN buffer, InGaN contact and active layers for A and B solar cell samples are shown in Figure 4(a). Where the FWHM measures for maximum half-width peaks; $\lambda$ and $\theta$ are the X-ray wave length and Bragg angle respectively. Meanwhile the plane tilt angles for $\mathrm{GaN}$ and $\mathrm{InGaN}$ are determined from the inclination of the lines seen in the Figure 4, the lateral mosaic sizes $L_{\|}$are obtained from the points which section the $y$ axis.

The lateral crystal sizes and tilt angles obtained from the W-H curves for the GaN buffer, InGaN active and contact layers are shown in the fourth and sixth columns of Table VI. The data related to the structural features of GaN are separated in the table with a comma from those of the InGaN layer and the " " symbol is used for the InGaN layers. The lateral crystal sizes and tilt angles for the GaN buffer layers in A and B InGaN solar cell samples provided the same values. This situation shows the same growing conditions at MOCVD for both samples. In comparison, the InGaN layers of both samples have lateral mosaic crystal sizes, and similar behavior like that of GaN. Furthermore, it is seen that the plane tilt angles of the second InGaN layers of samples had the same value. In addition to this similarity, there are some differences determined between the mosaic parameters of these InGaN layers. The lateral mosaic crystal length of the second InGaN layer and the plane tilt angle in the first InGaN layer of sample A had a lower value than those of the same layers in sample B. when the tilt in the graded structure is compared with non-grade structure, a significant difference does not exist. The reason for this is because the insertion of graded interlayers has more effect on twist than the tilt. ${ }^{24,25}$

Furthermore, this result had a negative effect on the crystal size, for it decreased the plane angle. When comparing the crystal sizes and the layer tilt angles of the interior InGaN layer of each sample, it was determined that the crystal sizes of the buffer layers were greater but more imperfect due to the higher plane tilt angle.

A vertical mosaic size and an $c$-axial combined strain in the radial scan direction of symmetric reflections cause an increase in FWHM values of the Bragg reflections. In the W-H graphic ${ }^{23}$ a counter graphic of FWHM $(\cos \theta) / \lambda$ versus $(\sin \theta) / \lambda$ is drawn for each reflection and a linear output is performed in Figure 4(b). The vertical mosaic dimensions $L_{\perp}$ are determined by Eq. (10) from the $y_{0}$ section point of the obtained line.

$$
L_{\perp}=0.9 /\left(2 y_{0}\right)
$$



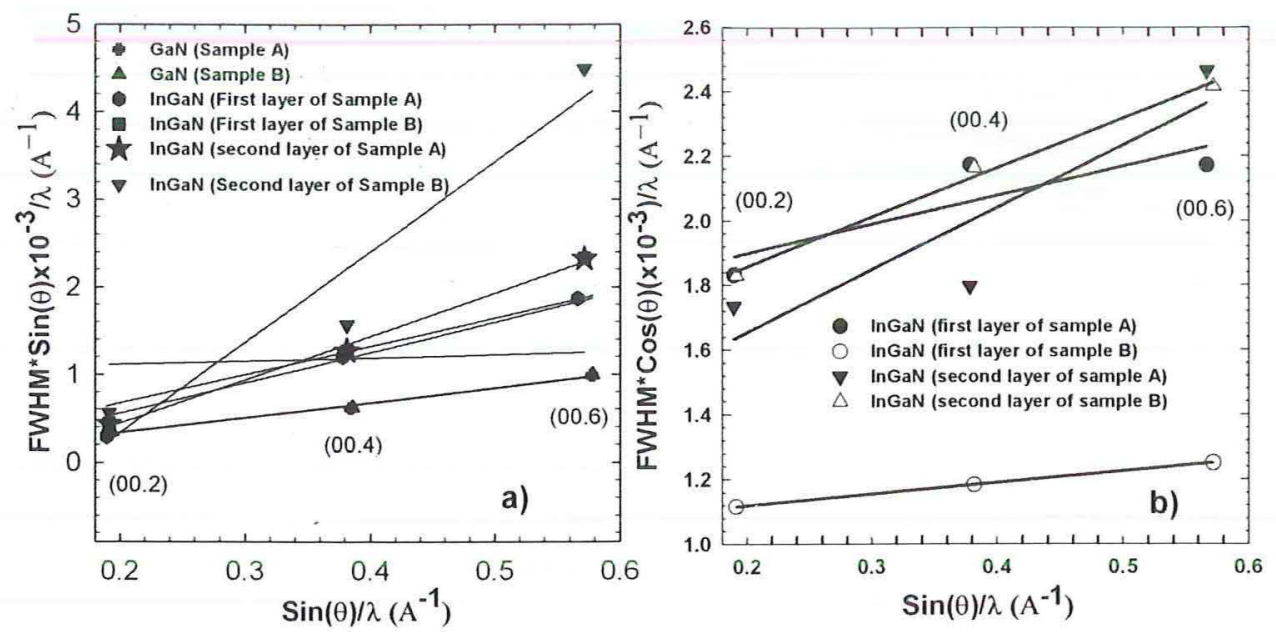

Fig. 4. Williamson-Hall graphics for the GaN, active and contact InGaN layers.

In the tilt of the obtained lines created from performing linear outputs by drawing FWHM $(\cos \theta) / \lambda$ versus $(\sin \theta) / \lambda$ for each reflection in the $\mathrm{W}-\mathrm{H}$ graphic are the determined heterogeneous strain values and the obtained vertical mosaic sizes regarding InGaN solar cell samples $\mathrm{A}$ and B (Table VI, column 3 and 5).

The vertical mosaic crystal size for the GaN layers of both samples provided the same experimental result, although the vertical mosaic crystal sizes for the $\mathrm{InGaN}$ layers have shown a different behavior. The vertical mosaic crystal sizes in the InGaN layers of the preliminary layer in sample A are lower than those of the Preliminary layer in sample B. However, this shows the exact opposite behavior of the collateral InGaN layer in samples A and B. Similarly, the same is observed on the preliminary and preliminary InGaN layers of samples A and B.

The vertical mosaic crystal size of the collateral preliminary of sample A was higher than that of the preliminary layer, the vertical mosaic crystal size of sample B's first layer was higher than that of the collateral layer (Table VI). The bulk of mosaic crystal size is due to the calibration trend of the MOCVD, though In contents of InGaN and layer thicknesses are different, yet this is related to the crystal quality of the layer.

The heterogeneous strains obtained for both samples are shown in the third column of Table VI. The heterogeneous strains of the $\mathrm{GaN}$ and $\mathrm{InGaN}$ layers of sample $\mathrm{A}$ are slightly higher than those of sample B. It is determined for the InGaN layer that the heterogeneous strains of the collateral layers of samples A and B are higher than those of the preliminary layers.

A method regarding the calculation of the edge and screw dislocation is based on the Burgers vectors as well as both the tilt angle and the lateral mosaic sizes. All types of dislocations are related to the mosaic crystal sizes, tilt angle and the twist angle. Metzger et al. ${ }^{26}$ stated that edge type dislocations with a mean twist angle Burgers vector $b=1 / 3\langle 11-20\rangle$ and screw type dislocations with a mean tilt angle Burger vector $b=\langle 0001\rangle$ (TDD) are related to each other. The intensity of the screw type dislocation density can be calculated by using Eq. (11). ${ }^{23} \cdot 27-30$

$$
N_{\text {screw }}=\frac{a_{\text {tilt }}^{2}}{\left|b_{\text {screw }}\right|^{2}}
$$

Here $a_{\text {tilt }}$ is a tilt angle for GaN and $b_{\text {screw }}=0.5185 \mathrm{~nm}$. The Burgers vector equals the lattice parameters. Therefore, the Burgers vector of the InGaN layers is calculated using Vegard's law. The $b_{\text {screw }}$ value of the InGaN layers for sample $A$ is determined to be $0.5240 \mathrm{~nm}$ and $0.5281 \mathrm{~nm}$, and for sample $B$ to be $0.5256 \mathrm{~nm}$ and $0.5315 \mathrm{~nm}$ respectively. Edge type dislocations convert consistently with the azimuthal rotation of the crystals around the surface normal by the Burgers vector

Table VI. Mosaic defect features of samples A and B.

\begin{tabular}{|c|c|c|c|c|c|c|c|}
\hline $\begin{array}{l}\text { Samples GaN } \\
\text { InGaN/InGaN }\end{array}$ & $\begin{array}{c}\text { In } \\
\text { Content } \\
(\%)\end{array}$ & $\begin{array}{c}\text { Heterogenous } \\
\text { Strain } \\
\left(\times 10^{-4}\right)\end{array}$ & $\begin{array}{l}\text { Lateral } \\
\text { mosaic } \\
\text { size }(\AA)\end{array}$ & $\begin{array}{l}\text { Vertical } \\
\text { mosaic } \\
\text { size }(\AA)\end{array}$ & $\begin{array}{c}\text { Tilt } \\
\text { angle } \\
\left(\times 10^{-3}\right)(\text { Deg. })\end{array}$ & $\begin{array}{c}\text { Screw TDD } \\
\left(\times 10^{4},\right. \\
\left.10^{5} / 10^{6}\right)\left(\mathrm{cm}^{-2}\right)\end{array}$ & $\begin{array}{c}\text { Edge TDD } \\
\left(\times 10^{6},\right. \\
\left.10^{7} / 10^{7}\right)\left(\mathrm{cm}^{-2}\right)\end{array}$ \\
\hline A (graded) & $10.5 / 18.4$ & $-3.75,2.25 / 4.75$ & $9000,900 / 250$ & $321,265 / 346$ & $1.70,4.2 / 1.03$ & $7.51,4.43 / 2.68$ & $5.50,0.806 / 290$ \\
\hline $\begin{array}{l}\text { B (non- } \\
\text { graded) }\end{array}$ & $13.6 / 24.9$ & $-4.00,1.0 / 3.75$ & $9000,900 / 265$ & $321,450 / 281$ & $1.70 / 5.0 / 1.03$ & $7.51,6.56 / 2.82$ & $5.56,1.06 / 361$ \\
\hline
\end{tabular}


$b=1 / 3\langle 11-20\rangle$. The edge type dislocation intensity can be calculated by the measured $a_{\text {twist }}$ twist angle. The edge type dislocation intensity can be calculated by Eq. (12) when the dislocations in the structure accumulate within the low angle grain boundaries. ${ }^{30.31}$

$$
N_{E}=\frac{a \phi}{2.1\left|b_{E}\right| L_{\|}}
$$

Where $a_{\phi}$ is the half-width peak of the asymmetric plane is related with twist. Burgers vector value is $0.3189 \mathrm{~nm}$ for $\mathrm{GaN}$, and $L_{\|}$is the lateral CL. For InGaN layers in sample $\mathrm{A} b_{E}$ are obtained as 0.3226 and $0.3253 \mathrm{~nm}$, and 0.3237 and $0.3276 \mathrm{~nm}$ for the layers in sample B. The edge and threading dislocations of samples $\mathrm{A}$ and $\mathrm{B}$ are obtained in Table I. The edge and threading dislocation values of the GaN layer in both samples have an almost equal value. It is seen that both the edge and the threading dislocations of the InGaN layers are lower in sample A. In previous presented studies, the insertion in the graded layer significantly reduces the edge and screw dislocation densities have been reported. ${ }^{24}$ It is observed that sample A has a more qualitative structure.

The FWHMs of $\omega$ and $\phi$ scans are determined by fitting to the Pseudo-Voigt. In this fitting, the FWHMs of $\omega$-scans increase due to scaling up of the $\chi$ angle and the FWHMs of $\phi$-scans decrease due to the increase of the $\chi$ angle. Besides, the $\omega$ and $\phi$-scans (12.1) coincide with each other at reflection $\chi^{31}$ at $78.6^{\circ}$. In fact, the $\chi$ angle reaches $90^{\circ}$ when the reflection plane is vertical to the sample surface. According to these results, the HRXRD rotating peak widths of $\omega$ or $\phi$ scans for this high $\chi$ angle are similar to their twist angles. In any case, the FWHMs of the $\phi$-scans are greater than those of the $\omega$-scans when a change in the $\chi$ angle occurs. Therefore, the mean twist angle of $\chi=78.6^{\circ}$ needs to be the mean value of the FWHMs of the $\omega$ and $\phi$ scans. The twist angles of $\mathrm{GaN}$ are determined as $0.118^{\circ}$ and $0.141^{\circ}$ for samples A and B respectively. ${ }^{32-34}$ It is seen that the value of sample B is slightly higher than that of sample A. For the InGaN layers, the image of the twist angle (12.1) was unable to be solved due to the reflection peaks of the plane remained in the background. ${ }^{35}$

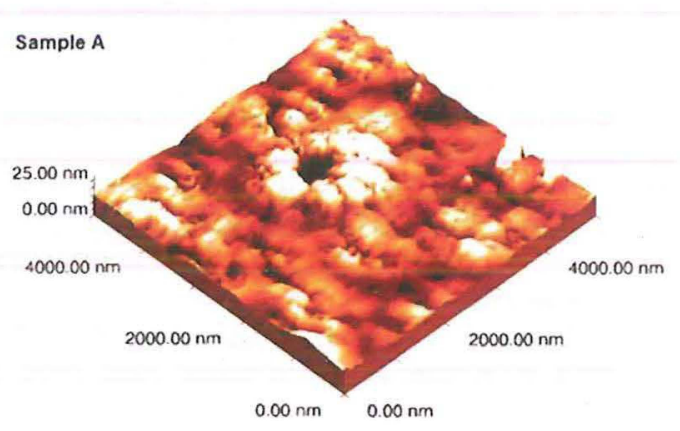

\subsection{AFM Observations}

The difference between the layers of multilayered structures is able to cause significant topographic changes on the sample surface. ${ }^{36}$ Therefore the surface features of the structures with InGaN layer of samples A and B are examined by AFM. For this purpose the surface images of the samples are obtained by using an Omicron VT STM/AFM device. The scans are performed on the surface in an area of $5 \times 5 \mu \mathrm{m}^{2}$. AFM images obtained by the scans conducted on the InGaN layers of the hetero-structures with InGaN layer of samples A and B (Fig. 5). It is brought to attention that there is a difference of the surface morphologies of the samples. Pits are seen on the surfaces of both samples. This is a typical situation for InGaN surfaces. However, meanwhile pit array are dominant on the surface of sample A, ordered hillock rows are dominant on the surface of sample B. Furthermore; the two-dimensional growth on the graded sample is more significant compared to the other. This is related to the diffusion of atoms.

On the other hand, roughness is a type of surface imperfection. There are many parameters used for the determination of the roughness. Root-mean-square (RMS) is the most widespread parameter used for the evaluation of the surface roughness. ${ }^{36.37}$ The RMS roughness values of samples A and B are measured to be 2.24 and 4.88 respectively. These values are determined to be coherent with the smooth surface of sample A, where the lateral growth was greater compared to the others. Chen et al. have reported segregation of indium next to vacancy islands or ordered vacancy rows on the surface. ${ }^{17.38}$ According to this, the rough surface of sample B with a higher indium percentage attributed to the segregation.

\subsection{JV Analysis}

Electrical performance of a solar cell could be determined by current density versus voltage $(J-V)$ measurements, which is the most common technique in photovoltaics. In value is inversely proportional to $V_{\text {oc }}$ in $\mathrm{InGaN}$ solar cell structures. Furthermore crystal imperfection in the structures changes the value of $V_{\mathrm{oc}}$ depending on changing of In concentration at low growing temperature. ${ }^{39}$ The $V_{\text {oc }}$ for the grown InGaN solar cells is typically $0.4 \mathrm{eV}$. According

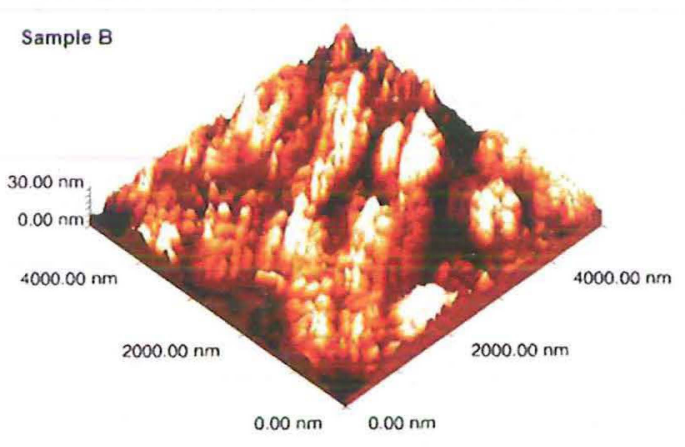

Fig. 5. AFM images of samples A and B. 
to the $V_{\mathrm{oc}}$ value, the solar cell performances of the graded sample A and sample B are $3.69 \%$ and $2.04 \%$, respectively. In the light of these results, it is determined that the solar efficiency of sample A has a higher performance. These results correspond with the other HRXD, AFM and $J V$ results. As a result, the continuous Indium flow indicates that the structural, morphological and electrical features of sample A are better than that of sample B. Graded InGaN structures have shown an enhanced performance in relaxing the strain in the $\mathrm{InGaN} / \mathrm{GaN}$ structures grown on sapphire, as compared to conventionally grown GaN structures. $^{40}$

\section{CONCLUSION}

In this study, we have studied the graded interlayers approach to improve the MOCVD grown InGaN epi-layer quality. By comparing In graded InGaN structure with non graded $\mathrm{InGaN}$ structure, we have found that the graded structure can improve the surface morphology and crystalline quality. The graded InGaN layer acts as a "transition layer" between InGaN epilayer and $\mathrm{GaN}$ template. It is demonstrated that the layer can release the strain and can significantly reduce tilt and the dislocations in the InGaN epilayer. Pits on the surfaces of both samples are seen in the AFM analysis. However, the pit array on the surface of In graded are dominant and the twodimensional growth is more distinct, compared to non graded. In non graded, the ordered hillock rows are dominant. Furthermore, the RMS roughness values of In graded and non graded are obtained as 2.24 and $4.88 \mathrm{~nm}$, respectively. These values show that In graded film is coherent with the smooth surface compared with the non graded one. According to the final conducted $J V$ calculations the solar battery performance of the graded samples A and B are determined to be $3.69 \%$ and $2.04 \%$ respectively. The experimental results show that graded growth of InGaN is a superior growth technique for high performance solar cell devices.

\section{References and Notes}

1. J. H. Ryou, W. Lee, J. Limb, D. Yoo, J. P. Liu, R. D. Dupuis, Z. H. Wu, A. M. Fischer, and F. A. Ponce, Appl. Phys. Lett. 92, 1 (2008).

2. H. Hamzaoui, A. S. Bouazzi, and B. Rezig, Sol. Energ. Mat. Sol. Cells 87, 595 (2005).

3. Q. Deng, X. Wang, H. Xiao, C. Wang, H. Yin, H. Chen, Q. Hou, D. Lin, J. Li, Z. Wang, and X. Hou, J. Phys. D, Appl. Phys. 44, 265103 (2011).

4. Z. Li, H. Xiao, X. Wang, C. Wang, Q. Deng, L. Jing, J. Ding, Z. Wang, and X. Hou, Chin. Phys. Lett. 30, 068402 (2013).

5. C. H. Chiu, D. W. Lin, Z. Y. Li, S. C. Ling, H. C. Kuo, T. C. Lu, S. C. Wang, W. T. Liao, T. Tanikawa, Y. Honda, M. Yamaguchi, and N. Sawaki, Proc. SPIE 7939, 79391 (2011).

6. N. C. Das, M. L. Reed, A. V. Sampath, H. Shen, M. Wraback, R. M. Farrell, M. Iza, S. C. Cruz, J. R. Lang, N. G. Young, Y. Terao, C. J. Neufeld, S. Keller, S. Nakamura, S. P. Denbaars, U. K. Mishra, and J. S. Speck, Journal of Electronic Materials 42 (2013).
7. M. Funato, M. Unde, Y. Kawakami, Y. Narukawa, T. Kosugi, M. Takanashi, and T. Mukai, Jpn. J. Appl. Phys. // 45, L659 (2006).

8. T. Lu, C. Kao, H. Kuo, G. Huang, and S. Wang, Appl. Phys. Lett. 92, 141102 (2008).

9. K. L. Lin, E. Y. Chang, Y. L. Hsiao, W. C. Huang, T. K. Li, D. Tweet, J. S. Maa, S. T. Hsu, and C. T. Lee, Appl. Phys. Lett. 91, 222111 (2007).

10. A. Krost, A. Dadgar, G. Strassburger, and R. Clos, Phys. Stat. Sol. (a) 200, 26 (2003).

11. Z. Ke-Bao, H. Miao, L. Ping-Yuan, W. Geng, L. Tai-Ping, and D. Chun-Hua, Chinese Physics B 22, 10 (2013).

12. C. E. Kendrick, Revisiting nitride semiconductors, epilayers, p-type doping and nanowires, Ph.D. Thesis, University of Canterbury, Canterbury, Christchurch (2008), pp. 63-72.

13. M. G. Suryanarayana, Norton, X-Ray Diffraction: A Practical Approach, Plenum Press, New York (1998).

14. C. Kisielowski, Semicon. Semimet. 57, 275 (1999).

15. G. Wassermann and J. Grewen, Texturen Metallischer Werkstoffe, Springer-Verlag, Berlin, Heidelberg (1962), pp. 754-5.

16. M. A. Moram and M. E. Vickers, Rep. Prog. Phys. 72, 036502 (2009).

17. Y. Baş, P. Demirel, N. Akın, C. Başköse, Y. Özen, B. Kınacı, M. K. Öztürk, S. Özçelik, and E. Özbay, Journal of Materials Science: Materials in Electronics 25, 3924 (2014).

18. M. Schuster, P. O. Gervais, B. Jobst, W. Hösler, R. Averbeck, H. Riechert, A. Iberlk, and R. Stömmerk, J. Phys. D: Appl. Phys. 32, 56 (1999).

19. S. I. Cho, K. Chang, and M. S. Kwon, J. Mater. Sci. 43, 406 (2008).

20. C. Kisielowski, J. Kruger, S. Ruvimov, T. Suski, J. W. Ager, E. Jones, Z. Liliental-Weber, M. Rubin, E. R. Weber, M. D. Bremser, and R. F. Davis, Phys. Rev. B 54, 17745 (1996).

21. V. Kachkanov, B. Leung, J. Song, Y. Zhang, M.-C. Tsai, G. Yuan, J. Han2, and K. P. O'Donnell, Scientific Reports 4, 4651 (2014).

22. V. S. Harutyunyan, A. P. Aivazyan, E. R. Weber, Y. Kim, Y. Park, and S. G. Subramanya, J. Phys. D: Appl. Phys. 34, A35 (2001).

23. G. K. Williamson and W. H. Hall, Acta Metall. 1, 22 (1953).

24. Md. Rafiqul Islam, Y. Ohmura, A. Hashimoto, A. Yamamoto, K. Kinoshita, and Y. Koji, Phys. Status Solidi C 7, 2097 (2010).

25. Md. Rafiqul Islam and Md. Arafat Hossain, IEEE/OSA/IAPR International Conference on Infonnatics, Electronics and Vision, ICIEV, Dhaka, Bangladesh (2012).

26. A. Hossain, R. Islam, M. K. Hossain, A. Hashimoto, and A. Yamamoto, Mater. Renew. Sustain Energy 10, 2 (2014).

27. O. Ambacher, Journal of Applied 31, 2653 (1998).

28. E. Arslan, M. K. Öztürk, Ö. Duygulu, A. A. Kaya, S. Özçelik, and E. Özbay, Applied Physics A-Materials Science and Processing 94, 73 (2009).

29. N. G. Weimann, L. F. Eastman, D. Doppalapudi, M. N. Hock, and T. D. Moustakas, J. Appl. Phys. 83, 3656 (1998).

30. M. E. Vickers, M. J. Kappers, R. Datta, C. McAleese, T. M. Smeeton, F. D. G. Rayment, and C. J. Humphreys, J. Phys. D: Appl. Phys. 38, A99 (2005).

31. X. H. Zheng, H. Chen, Z. B. Yan, Y. J. Han, H. B. Yu, D. S. Li, Q. Huang, and J. M. Zhou, J. Cryst. Growth 255, 63 (2003).

32. S. C. Jain, M. Willander, J. Narayan, and R. V. Overstraeten, J. Appl. Phys. 87, 965 (2000).

33. O. Brandt, Y. J. Sun, H. P. Schönherr, K. H. Ploog, P. Waltereit, S. H. Lim, and J. S. Speck, Appl. Phys. Lett. 83, 90 (2003).

34. J. K. Sheu, IEEE Electron Device Letters 30, 225 (2009). 
35. P. Gay, P. B. Hirsch, and A. Kelly, Acta Metall. 1, 315 (1953).

36. S. Çörekçi, M.K. Öztürk, M. Çakmak, S. Özçelik, and E. Özbay, Mater. Sci. Semicond. Process. 15, 32 (2012)

37. S. Çörekci, M. K. Öztürk, A. Bengi, M. Çakmak, S. Özçelik, and E. Özbay, J. Mater. Sci. 46, 1606 (2011)

38. T. Tao, Z. Zhao, L. Lian, S. Hui, X. Zili, Z. Rong, L. Bin, $X$. Xiangqian, L. Yi, H. Ping, S. Yi, and Z. Youdou, Journal of Semiconductors 32, 1 (2011).
39. O. Jani, P. Mahala, S. K. Behura, A. Ray, and C. Dhanavantri, The effect of indium composition on open-circuit voltage of InGaN thin-film solar cell: An analytical and computer simulation study, AIP Conf. Proc., Mumbai, India (2012), Vol. 1451.

40. T. L. Song, S. J. Chua, and E. A. Fitzgerald, Graded InGaN Buffers for Strain Relaxation in GaN/InGaN Epliayers Grown on Sapphire, DSpace@MIT: Massachusetts Institute of Technology (2002). 